\title{
Simulación multimedia interactiva para el logro del aprendizaje procedimental del manejo del microscopio
}

\section{Resumfen}

Se llevó a cabo una investigación para comparar la efectividad de dos estrategias instruccionales para el logro del aprendizaje procedimental: una basada en el uso de una secuencia instruccional siguiendo cinco principios de aprendizaje de Merrill, y la otra, basada en el USO de secuencias autónomas. Adicional mente, se determinó la manera como los estudiantes interactúan con las actividades de instrucción en relación con su estilo preferencial de aprendizaje, Șe utilizó un diseño experimental entre dos grupos, con postprueba únicamente con estudiantes en una universidad de educacion a distancia. Los resul tados mostraron que la secuencia de aprendizaje basada en los principios de aprendizaje de M errill fue más efectiva para el logro del aprendizaje procedimental en la dimensión conocimiento declarativo y en el indicador tiempo de aprendizaje, aunque no mostró efecto significativo sobre el indicador precision en la destreza tecnica. No hubo diferencia estadistica entre los estilos de aprendizaje y ladedicación de tiempo a la actividad preferencial de aprendizaje, pero s! hubo tendencias diferentes en la selección de actividades de instrucción. Los resultados mostraron que la simulación multimedia utilizada se adaptó a los cuatro estilos de aprendizaje.

Palabras clave: Simulación, aprendizaje experiencial, aprendizaje multimedia, estilos cognitivos, material multimedia. 


\title{
Interactive multimedia simulation to succeed in learning microscope use procedures
}

\begin{abstract}
The purpose of this study was to compare the effectiveness of two instructional strategies to get procedural knowledge: one based on M errill's learning principles, and the other based on independent instructional sequences. Additionally, the way students interact with instructional activities regarding their preferred learning style was determined. A n experimental design between two groups was used, applying a post-test only among students from a distance learning college. Findings showed the instructional sequencebased on M errill's learning principles was more effective in achieving procedural learning in its declarative knowledge dimension and in the time of learning indicator, but showed no significant effect on accuracy in the skill technique indicator. There was no statistical difference between learning styles and time commitment to the preferential learning activity, but there were different trends in the selection of instructional activities. Findings showed that the multimedia simulation used was adapted to the four learning styles.
\end{abstract}

Keywords: Simulation, experiential learning, multimedia instruction, cognitive style, multimedia materials.

Recibido: 21 Enero de 2011 Aceptado: 3 Junio de 2011

\section{Introducción}

El problema centrado en la búsqueda de estrategias de instrucción efectivas para el aprendizaje procedimental a distancia es un problema de naturaleza aplicada y surgio en la universidad donde se llevo a cabo este estudio, a partir de los datos estadisticos del ano 2010 de la Oficina de Registro y Control A cademico (Universidad Nacional A biertay a Distancia [UNA D], 2010), Los datos mostraron que el 37\% de los estudiantes virtuales del curso de biologia, por razones geograficas, economicas, de discapacidad o de otra indole no pudieron asistir a las practicas de laboratorio tradicional para real izar el aprendizaje procedimental de manejo del microscopio optico, problema que afecta su avance académico. 
Revista de

investigaciones UNAD

Volumen 10. Número 1. Junio 2011

La literatura muestra que las simulaciones representan una alternativa instruccional efectiva para el aprendizaje procedimental (Hardy, 2008). A dicionalmente, facil itan y disminuyen el costo de la logistica academica en relacion con tiempo, infraestructura y seguridad para los estudiantes (Slabicki, 2007).

Un antecedenteimportanteen el aprendizajeprocedimental es la implementación de la microscopia virtual para examenes de certificacion profesional en patologia médica (Weinstein, 2005). Hacia el año 2000 entró en progresivo desuso la microscopia tradicional en la educacion medica y se fortalecio la microscopia virtual mediante simuladores; segun las encuestas, el 50\% de los cursos de patologia ya tienen o esperan tener microscopia digital (Dee, 2009).

Davidson (2000) señaló la necesidad por parte de los instructores de utilizar las estrategias de instruccion que se articul en mejor a los estil os de aprendizaje para alcanzar mejores resultados. Davidson (2000) y White y Sivitanides (2002) reportaron el logro de mejores resul tados de aprendizaje procedimental cuando se adecua la estrategia de instrucción a los estilos preferenciales de aprendizaje de los estudiantes.

Dada la importancia y la necesidad de entender cómo utilizar efectivamente las simulaciones interactivas para el logro del aprendizaje procedimental del manejo del microscopio en ambientes virtuales, considerando los estilos de aprendizaje, se planteo el proposito de la investigacion como de caracter dual: a) determinar la efectividad de dos estrategias de instruccion para aprendizaje procedimental a distancia en campus virtual, con la finalidad de suministrar una experiencia equival ente de aprendizaje respecto al laboratorio tradicional, y b) determinar como interactuan los estudiantes con las actividades de instruccion en relación con su estilo preferencial de aprendizaje para el logro del aprendizaje procedimental.

Como marco teórico en este estudio se utilizaron cinco principios de aprendizaje de M errill. (centralización en una tarea, activación de conocimientos previos, demostracion del procedimiento aplicacion mediante ejercitacion eintegración del conocimiento y de la habilidad adquirida a la vida real o cotidiana del estudiante). Este conjunto de actividades de instrucción se denomina también ciclo de aprendizaje (M errill, 2007), Como referencia a las prequntas de investigacion se tomaron las hipotesis planteadas por M errill (2007): a), el aprendizaje a partir de un, programa instruccional se facilitara en proporcion directa a la implementación de los primeros,principios de instrucción, y b) el aprendizaje a partir de un programa instruccional se favorecera en proporcion directa al grado en que los primeros principios se utili icen en la secuencia exacta, en lugar de ser implementados al azar. A dicionalmente, la teoria multimediá de Mayer (2010) oriento el diseno instruccional de la simulacion multimedia interactiva utilizada en esta investigacion. 
Carmen Eugenia Piña López. Ed. D. y Anymir Orellana. Ed. D.

Simulación multimedia interactiva para el logro del aprendizaje procedimental del manejo del microscopio,

artículo producto de la investigación

En microscopía, la estrategia instruccional basada en simulación multimedia interactiva representa para los estudiantes a distancia, específicamente, una experiencia de aprendizaje equivalente al aprendizaje tradicional en el laboratorio, según la teoría de equival encia de Simonson, Smaldino, A lbright, y Zvacek (2006).

Se espera que los hallazgos de la investigación contribuyan a los avances investigativos sobre la efectividad del uso de los simuladores como estrategla instruccional para el logro del aprendizaje procedimental en estudiantes a distancia que, a su vez, aporten a una educación más incluyente en relación con su cobertura a estudiantes marginados de la posibilidad de continuar sus estudios superiores por barreras geograficas o socioeconomicas.

\section{Metodología}

Para lograr el propósito dual del estudio se plantearon dos preguntas: ¿Existe diferencia significativa en el logro del aprendizaje procedimental del manejo del microscopio con simulación multimedia entre el grupo que utiliza una estrategia de instruccion que sigue la secuencia de los primeros principios de aprendizaje de Merrill (2007) y el grupo que sigue una secuencia autonoma? ¿Existe diferencia significativa en la forma como los estudiantes interactúan con las actividades de instrucción entre los distintos estilos de aprendizaje?

Para responder a las preguntas se llevó a cabo un experimento de comparación entre dos grupos de manera al eatoria con postprueba unicamente. El ambiente instruccional del experimento correspondio a una simulación multimedia que permitio al participante interactuar con un microscopio virtual compuesto de módulos de navegación basados en los cinco principios de instruccion de M errill, (2007) para lograr el aprendizaje procedimental con una comprension simultanea de conceptos y principios de microscopia optica.

La variable independiente fue la estrategia de instrucción con dos niveles: El Grupo A, al quesel eaplico la secuencia de los primeros principios de instruccion de M errill en las actividades de instruccion para el aprendizaje procedimental de manejo del microscopio; y G rupo B que hizo una exploracion y selección autonoma de las actividades de instruccion para el aprendizaje procedimental de manejo del microscopio.

La variable dependiente fue el aprendizaje procedimental con dos dimensiones: conocimiento declarativo sobre microscopla, y destreza tecnica de manejo del microscopio Se plantearon dos indicadores: la precision en la ejecucion de los pasos procedimentales y el tiempo invertido en la ejecucion del procedimiento. 
Revista de

investigaciones UNAD

Volumen 10. Número 1. Junio 2011

Procedimiento. La Figura 1 muestra el procedimiento de investigación:

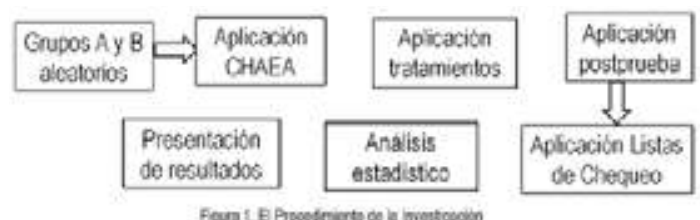

Figura 1. El procedimiento de investigación

1. Asignación aleatoria de los participantes. Al inicio de la sesión experimental mediante extraccion al eatoria de fichas alfanuméricas se realizo la asignación de los 80 participantes del curso de biologia a los grupos de tratamiento. El codigo alfabetico de cada ficha fue A O B e indico el grupo de tratamiento, mientras el código numérico de 1 a 40 por cada código alfabético identifico al estudiante dentro de un grupo y en un computador especifico, manteniendo su anonimato para el proceso de recoleccion de datos. El codigo numerico fue utilizado por el participante cada vez que respondia a un instrumento. Todas las interacciones del estudiante durante el experimento fueron registradas mediante pantallas capturadas con el software Topviewsoft de uso libre y.previamente instalado en cada computador. El software Topviewsoft esta di sponible para su descarga en el sitio http://www.topviewsoft.com/free-screen-video-capture.html.

2. Administración del cuestionario CHAEA. Para determinar el estillo de aprendizaje, los estudiantes procedieron a responder el cuestionario CHAEA de A lonso y Gallego (2008). L a administracion de este instrumento se hizo en linea desde la direccion http://www, estilosdeaprendizaje.es/chaea/chaea.htm. El tiempo de duración fue de 15 minutos. El resultado del estilo preferencial de aprendizaje se genero automáticamente. La preferencia del participante se determino con un baremo construido con los datos de la poblacion participante siguiendo el modelo de Alonso, Gallego y Honey (1999).

3. Aplicación de los tratamientos. El tiempo de aplicación de las estrategias instruccionales fue de 45 minutos y, se realizo en el siguiente orden: a) el participante leyó la guía de instruccion impresa correspondiente a su grupo e inició la interacción con la simulación. La guía del grupo A entrego instrucciones obligatorias sobre el uso de la secuencia de los primeros principios de instruccion de M errill, accesible mediante botones de navegacion del menu de la simulación multimedia. La gula del Grupo B señalo la autonomia de los participantes para explorar y seleccionar a traves del menu de la simulacion as acțividades de instruccion para el aprendizaje procedimental de manejo del microscopio. El software Topviewsoft genero un video donde quedaron 
Carmen Eugenia Piña López. Ed. D. y Anymir Orellana. Ed. D.

Simulación multimedia interactiva para el logro del aprendizaje procedimental del manejo del microscopio,

artículo producto de la investigación

registradas todas las acciones que el participante realizó, la secuencia de actividades de instruccion seguidas, el tiempo que los participantes invirtieron en cada una de las actividades y el numero de aciertos en el manejo simulado del microscopio. Al terminar la interaccion con la simulacion, cada estudiante guardó la grabacion del video con el código alfanumerico confidencial. Este proceso fue supervisado por los docentes administradores del experimento con el fin de garantizar la grabacion del proceso para la recolección de la data.

4. Administración de la postprueba, Esta se aplicó a los dos grupos en medio impreso inmediatamente después de la interacción del participante con las actividades de la estrategia instruccional, con el fin de medir el conocimiento declarativo. El tiempo para responder la postprueba fue de 30 minutos. El participante respondio el cuestionario de la postprueba de manera anónima, identificando su postprueba con el codigo alfanumęrico confidencial que extrajo al eatoriamente cuando ingreso al lugar del experimento. La identificacion de la postprueba con el código permitio confrontar los resultados del aprendizaje declarativo con los resul tados de la destreza técnica y del cuestionario de estilo de aprendizaje identificados con el mismo codigo.

Al finalizar el experimento, los docentes responsables de la administración de los instrumentos transfirieron los result tados del cuestionario a una hoja de calculo de Excel para ingreso al paquete estadístico SPSS 17 destinado a su posterior análisis estadistico.

5. Análisis de los datos. LOS datos resultantes de la aplicación de los instrumentos se analizaron con estadistica descriptiva y parametrica, Para el análisis estadístico se utilizó una version trial del paquete estadístico SPSS 17, fabricado por IBM en Estados U nidos. El análisis de datos se realizo con un nivel de significancia de valor $p<0,05$. El primer paso en el análisis de datos utilizo estadistica descriptiva para determinar las trecuencias y el porcentale de estudiantes que contestaron los instrumentos. En cada grupo se calculo la frecuencia y el porcentaje de participantes del género masculino y, femenino. I gualmente se calculo la media, la mediana, la moda, la desviación estandar y los límites mínimo y máximo en cuanto a la edad de los participantes. En relación con los estil os de aprendizaje, se cal culó para cada grupo la frecuencia y el porcentaje de cada uno de los cuatro estilos. El segundo paso fue el analisis de datos con estadistica inferencial para cada una de las preguntas.

Para responder la primera pregunta, ¿Existe diferencia significativa en el logro del aprendizaje procedimental del manejo del microscopio con simulacion multimedia entre el grupo que utiliza una estrategia de instruccion que sigue la secuencia de los cinco principios de aprendizaje de M errill (2007) y el grupo que sigue una secuencia autonoma?, se utilizo la prueba t de Student de dos col as, que comparo las medias de los puntajes de la postprueba, del numero de aciertos y del tiempo invertido en el manejo del microscopio. 
Revista de

investigaciones UNAD

Volumen 10. Número 1. Junio 2011

Para responder la segunda pregunta, ¿Existe diferencia significativa en la forma como los estudiantes interactuan con las actividades de instruccion entre los distintos estilos de aprendizaj e?, se utilizo un analisis de varianza A N OVA de una via, que puede comparar mas de dos grupos y permite identificar si hay diferencia significativa entre las medias de los grupos.

En este estudio, los factores categóricos fueron los estilos de aprendizaje y el tipo de actividad de instruccion. Estos dos factores definieron los grupos cuyas medias fueron comparadas. Los, estilos de aprendizaje se denominaron asl: $A=A$ ctivo; $R=$ Reflexivo; T = Teórico y $P=$ Pragmático. Las actividades de inștrucción se denominaron: $F=$ Fundamentación; $D=$ Demostración; $E=$ Ejercitacion y $\mathrm{C}=$ Componentes.

La variable dependiente fue la forma como los estilos de aprendizaje interactuaron con una de las cuatro actividades de instruccion. Los indicadores para esta medicion fueron: a) el mayor porcentaje de tiempo extra dedicado a una actividad en relación con las demás actividaades y bo) la mayor frecuencia con que los participantes de cada estil o prefirieron seleccionar una de las cuatro actividades de instruccion.

Como el factor denominado actividad de aprendizaje causó efecto estadístico altamente significativo en el tiempo extra de dedicacion preferencial, se aplico poșt hoc, la prueba de rango múltiple de Duncan. Ésta permitio la comparacion de las medias de los tratamientos, todos contra todos, de manera que cualquier diferencia existente entre un tratamiento y otro, se vera reflejado en este análisis,s. Esta prueba utiliza un nivel de significancia variable que depende del numero de medias que entran en cada etapa de comparacion. La idea es que a medida que el numero de medias aumenta, la probabilidad de que se asemejen disminuya.

\section{Resultados}

Todos los ochenta participantes culminaron las actividades del experimento. El 80\% fueron mujeres y la edad promedio fue de 33 años. Se realizaron las pruebas estadísticas a un nivel de significancia de 0.05. A continuación se presentan y discuten los resultados segun las preguntas de investigacion.

Pregunta 1 para el indicador conocimiento declarativo

Los resultados de la prueba $t$ para muestras independientes, $t=2.945$ y $p<0.05$, de la postprueba sobre conocimiento declarativo, indicaron superior desempeño en el Grupo A, que utilizó la secuencia de M errill, en comparacion con el $G$ rupo B que usó secuencias autonomas. Se comprobaron 
Carmen Eugenia Piña López. Ed. D. y Anymir Orellana. Ed. D.

Simulación multimedia interactiva para el logro del aprendizaje procedimental del manejo del microscopio,

artículo producto de la investigación

las dos hipótesis de Merrill: al el aprendizaje a partir de un programa instruccional se facilitará en proporcion directa a la implementacion de los primeros principios de instrucción, y b) el aprendizale a partir de un programa instruccional se favorecera en proporcion directa al grado en que los primeros principios se utilicen en la secuencia exacta, en lugar de ser implementados al azar. Este resultado se encontro compatible con investigaciones previas de Mampadi, Chen, Ghinea y Chen (2011), quienes encontraron diferencia significativa en el logro del aprendizaje declarativo medido con una postprueba, entre un grupo de estudiantes que utilizo como estrategia instruccional uná hipermedia adaptada al estilo de aprendizaje y otro grupo que utillizó una hipermedia sin adaptacion al estilo. Este resul tado también concuerda con hallazgos de Garcia, Perez y Talaya (2008) sobre la preferencia estudiantil por métodos direccionados.

El resultado obtenido en este estudio no coincide con los resultados reportados por Davidson.(2000), quien no encontro diferencia significativa en el logro del conocimiento declarativo aplicado al manejo de una hoja de cálculo entre dos metodos de ensenanza: una practica guilada con unos pasos secuenciales especificos y una practica supervisada con autonomia para navegar libremente.

Un factor que puede explicar esta diferencia de resultados es que el conocimiento declarativo fue, evaluado por Davidson (2000) con una postprueba que se aplico después de varias semanas de aprendizaje del curso académico, mi entras que en esta investigacion la adquisicion del conocimiento declarativo formo parte de las actividades de instruccion desarrolladas por los participantes en una sola sesión de trabajo y fue evaluada con una postprueba al finalizar la sesion. Queda como recomendacion para futuras investigaciones, evaluar la retención del conocimiento declarativo a corto y mediano plazo en procesos de instrucción con actividades de aprendizaje secuenciales y de navegacion autonoma.

Pregunta 1 para el indicador aciertos en el manejo del microscopio en la destreza técnica

Al comparar las medias del número de aciertos en el manejo del microscopjo se obtuvo $t=1,602, p>0,05$. N o hubo diferencia significativa en la precision de manejo del microscopio, medida por el numero de aciertos. Este resul tado es compatible con los hall azgos de Pratt (2000), quien encontro que no hubo diferencia significativa en la precision durante el manejo de un software. Este hall azgo tambien coincide con el reportado por Davidson (2000), quien encontro que tanto las practicas quiadas como la práctica supervisada, produjeron similares resul tados de rendimiento de los estudiantes en relacion con la habilidad procedimental medida por los pasos realizados con acierto al manejar las hojas de calculo. 
Revista de

investigaciones UNAD

Volumen 10. Número 1. Junio 2011

Pregunta 1 para el indicador tiempo de manejo del microscopio en la destreza técnica

Al comparar las medias entre los grupos, se halló diferencia significativa t $=-5.814$, y $p<0.05$, con menor tiempo invertido en el manejo simulado del microscopio por parte del grupo que utilizo la secuencia de los primeros principios de aprendizaje deM errill. Es importante destacar que el grupo que siguio la secuencia de aprendizaje de M errill tambien obtuvo un promedio en la calificación de la postprueba superior al grupo que selecciono una secuencia autonoma. Este resul tado explicaria la mayor eficiencia en el uso del tiempo, atribuible a que el conocimiento declarativo logrado genero mayor seguridad en la toma de decisiones correspondientes a cada paso y a un menor numero de repeticiones requeridas durante la ejecucion del procedimiento.

La mayor habilidad procedimental evidenciada por la reducción en el tiempo de manejo del microscopio, es coherente, además, con el hallazgo de L ewis (2008), quien encontro una relación inversa según la cual a menor tiempo en la ejecucion procedimental, corresponde mayor precision en la habilidad procedimental. Otra posible explicacion para la mayor destreza tẹcnica obtenida en el Grupo A que gastó menor tiempo de ejecución, se deriva de los hall azgos de M errill (2007), segun los cual es para el logro de la destreza procedimental, los ejemplos trabajados con una secuencia instruccional basada en submetas, optimizan la habilidad del aprendiz debido a que le exigen menor esfuerzo mental. Merrill. (2007) encontró este resultado consistente con la teoria de la carga cognitiva.

En síntesis, de los tres componentes que miden el aprendizaje procedimental, la secuencia de Merrill podría ser predictora de mejor desempeño en dos componentes, uno de conocimiento declarativo y otro de destreza técnica referida al tiempo de ejecución, lo cual indico una mayor capacidad de eficiencia procedimental, En cambio, no es predictora en el numero de aciertos durante el procedimiento, que es el segundo aspecto de la destreza tecnica, Se sugiere la conveniencia de adoptar la secuencia de M errill en el diseño instruccional de simulaciones destinadas a apoyar aprendizajes procedimentales.

Pregunta 2 para determinar diferencia en la forma como los estudiantes interactúan con las actividades de instrucción según los distintos estilos de aprendizaje

Los resultados de la prueba estadística AN OVA de una vía, $F=1.088$, y $p$ $>0.05$, permitieron concluir que el porcentaje de tiempo extra dedicado a la actividad preferencial de instruccion no presentó diferencia significativa 
Carmen Eugenia Piña López. Ed. D. y Anymir Orellana. Ed. D.

Simulación multimedia interactiva para el logro del aprendizaje procedimental del manejo del microscopio,

artículo producto de la investigación

entre los estilos de aprendizaje. Estos resultados son compatibles con los hall azgos encontrados por Reese y Tabachnik (2010), quienes no encontraron diferencia significativa entre los diversos estilos de jugadores al evaluar el tiempo dedicado al aprendizaje procedimental en juegos por computador con actividades de instruccion. Los investigadores comproparon que en este tipo de experiencia hubo dos tases de aprendizaje, una primera, de preaprendizaje durante la cual el avance de los participantes fue muy lento y diferenciado individual mente porque aún los jugadores no habían llegado al nivel exacto del logro del aprendizaje procedimental. En la segunda fase, de post-aprendizaje, todos los jugadores avanzaron de manera rapida y homogenea hacia la meta procedimental del juego. Estos resultados sucieren que en este estudio el tiempo extra dedicado a la actividad preferencia por los diversos estilos de aprendizaje ocurrió cuando ya los participantes habían logrado el aprendizaje, razon por la cual el uso de tiempo extra no era una necesidad cognitiva fuerte.

De manera Similar, los resultados coinciden con los reportados por L o y Chan (2008) en una investigación que midio cada cinco minutos la forma como los diversos estilos de aprendizaje navegaron entre páginas web instruccionales. Ellos encontraron que durante los primeros 25 minutos no fue posible detectar diferencias estadisticas en el tiempo extra de dedicacion en los contenidos instruccionales. Sin embargo, entre los 26 y 30 minutos hallaron diferencias significativas entre dos estilos de aprendizaje en el tiempo, extra de permanencia en los contenidos instruccionales preferenciales, situación que en esta investigación no se presento debido al que el tiempo requerido para desarrollar cualquier actividad preferencial fue inferior a diez minutos. Por el contrario, en un estudio que investigo la relación de los estillos de aprendizaje dependiente e independiente de campo y su comportamiento durante una actividad instruccional asistida por hipermedia, Liu y Reed (1994) encontraron que los dependientes de campo gastaron mas tiempo en la interaccion con el curso, accedieron mas veces al mismo y observaron más veces los videoclips.

Con el fin de determinar si los valores del porcentaje de tiempo extra dedicado a la actividad preferencial dependian de uno de estos dos factores: el estilo de aprendizaje 0 el tipo de actividad y la interacción entre ambos, se realizo un A nálisis U nivariado AN OVA de dos factores. L OS datos evidenciaron que el factor estilo, con los valores $F=0.914$ y $p>0.05$, no afecto el tiempo de dedicación preferencial a la actividad. Por el contrario, el factor denominado tipo de actividad de aprendizaje, $F=5.481$ y val or $p<0.05$, si mostro un efecto altamente significativo en el tiempo extra de dedicacion preferencial.

Para determinar la relación entre la variable estilo de aprendizaje y el tipo de actividad seleccionada, se elaboro una tabla de contingencia en la cual se cruzaron las variables, estil os de aprendizaje y actividades de instruccion. LoS resultados mostraron las siguientes preferencias de seleccion: para el estilo 
Revista de

investigaciones UNAD

Volumen 10. Número 1. Junio 2011

activo, la actividad de Ejercitación fue seleccionada como preferencial por el mayor porcentaje de participantes, correspondiente a un 61:1\%; para el estilo reflexivo, las actividades Ejercitacion y Fundamentacion fueron seleccionadas por iqual por el 44.4\% de los partici pantes; para el estil o teorico, 41.4\% prefirio mayoritariamente la actividad de Fundamentacion; asi mismo, los participantes del estilo pragmatico mostraron preferencia mayoritaria con un $53.3 \%$ por la actividad de Ejercitacion.

En la Figura 2 se puede visualizar qué actividad al canzó la máxima preferencia dentro de cada estilo de aprendizaje. Se destaca que el estilo reflexivo no mostró ninguna preferencia por la actividad de Demostración. I gualmente el estilo pragmatico no tuvo ninguna preferencia por la actividad Componentes. Se corroboró la Ejercitación como la tendencia preferencial de mayor a menor en las actividades.

Fundamentación, componentes y demostración

Forma de interacción de los participantes con las actividades de instrucción

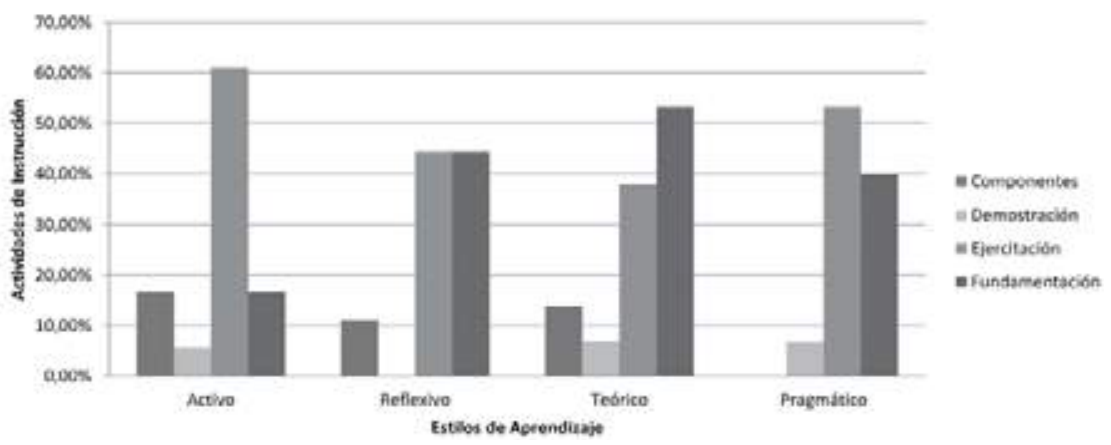

Figura 2. Forma de interacción de los participantes con las actividades de instrucción

Al responder la segunda pregunta se encontró adicional mente una tendencja a seleccionar, preferencialmente, en las actividades de instruccion segun el estilo de aprendizaje, la actividad de Ejercitacion en los estilos activo y pragmático, mientras que en el estilo teorico predominó la preferencia por la actividad de Fundamentacion y en el estillo reflexivo la preferencia fue la actividad de Fundamentacion y de Ejercitacion. Estos hal lazgos coinciden con la caracterización que Alonso, Gallego y Honey (1999) plantearon para cada esti|lo de aprendizaje determinado con el cuestionario CHA EA: Las personas de estilo activo tienden hacia lo novedoso; los refl exivos revisan cuidadosamente todos los contenidos antes de tomar decisiones, los teoricos se inclinan a 
Carmen Eugenia Piña López. Ed. D. y Anymir Orellana. Ed. D.

Simulación multimedia interactiva para el logro del aprendizaje procedimental del manejo del microscopio,

artículo producto de la investigación

articular o integrar ideas, teorías y sistemas de pensamiento; los pragmáticos buscan aplicaciones utiles y realistas de los nuevos contenidos.

De esta manera, los participantes con estilo activo prefirieron la actividad novedosa de Ejercitacion que ofrecio la nueva experiencia de manejo simulado del microscopio. Los participantes de estilo pragmático tambien prefirieron la Ejercitación, que es una forma de comprobar la funcionalidad 0 la efectividad real de la aplicación de simulación, Los participantes del estil o teorico escogieron la actividad de Fundamentación, que es precisamente la base instruccional que aporta una integracion teórica de los principios de la microscopia que explican o sustentan los pasos requeridos al manejar un microscopio desde el punto de vista de las leves físicas de la optica. En cambio, los estudiantes con estillo reflexivo se dedicaron por igual a las actividades de Fundamentacion y de Ejercitación, es decir, revisaron cuidadosamente tanto lo teorico como lo práctico para asegurar su aprendizaje.

El hallazgo en esta investigación sobre las tendencias a una actividad según el estil o de aprendizaje, es consistente con estudios previos como, los de Lo y Chan (2008), cuyos resul tados de investigacion mostraron que había diferencia significativa en el comportamiento selectivo de navegacion entre aprendices con diferentes estilos cognitivos, y que los aprendices de cada estilo tenian una preferencia personal por determinados componentes instruccionales.

\section{Conclusiones}

En el marco del propósito dual de la investigación, los resultados indicaron: a) La secuencia de M errill (2007) favorecio el aprendizaje procedimental; b) la simulacion multimedia utilizada se adapto a los cuatro estilos de aprendizaje; c) el logro del aprendizaje depende directamente de la estrategia instruccional utilizada; d) mediante la interacción con el simulador, los estudiantes pueden lograr una experiencia equivalente de aprendizaje con relacion al aprendizaje de microscopia en laboratorios presenciales; e) el diseño instruccional apoyado en los primeros principios de aprendizaje de Merrill y en la teoria del aprendizaje multimedia de Mayer contribuye efectivamente al aprendizaje.

Estas conclusiones confirman a su vez el planteamiento de Clark (2001) cuando señalo que los medios y sus atributos influyen de manera importante en el costo o en la velocidad del aprendizaje pero que solamente el uso de métodos instruccional es adecuados influira en el aprendizaje. En estẹ sentido, la simulacion multimedia interactiva para el manejo del microscopio optico es un medio que tacilita la gestion educativa, y la estrategia instruccional incorporada es el factor de mejoramiento del aprendizaje. 
Revista de investigaciones UNAD

Volumen 10. Número 1. Junio 2011

Una reflexión adicional es que el uso de secuencias instruccionales que el docentey el diseñador instruccional presentan como óptimas parael aprendizaje, se afianzan por lo general en principios validados de aprendizaje en amplios circulos academicos. Por esta razon, en el proyecto se encontro logico otro resultado aun no comentado: Los estudiantes del grupo experimental que tenian libertad para escoger una secuencia instruccional autonoma o personal, en un porcentaje significativo aunque no preponderante, seleccionaron coincidencialmente la misma secuencia de M errill. Estos hall azgos parecen indicar que intuitivamente los estudiantes pueden preferir secuencias instruccional es acordes con principios validados de aprendizaje.

\section{Referencias bibliográficas}

Alonso, C., Gallego, D. \& Honey, P. (1999). Los estilos de aprendizaje. Procedimientos de diagnóstico y mejora. Bilbao: Ediciones Mensajero.

Alonso, C. \& Gallego D. (2008). Cuestionario Honey-Alonso de Estilos de Aprendizaje [en línea]. Madrid: Recuperado de: ttp://www .estilosdeaprendizaje.es/chaea/chaea.htm

Clark, R. E. (2001). New directions: Evaluating distance education technologies. En R. E.

Clark (Ed.), Learning from media: Arguments, analysis and evidence. (pp. 299-317). Greenwich, CT: Information Age Publishing.

Davidson, C. (2000). Comparative analysis of teaching methods and learning styles in a high school computer spreadsheet unit. (Disertación Doctoral, Universidad de M issouri, Columbia 2000). Recuperado el 13 de agosto de 2010, de ProQuest Dissertations \& Theses. http://ezproxylocal. library.nova.edu/docview/304611503?accountid $=6579$.

Dee, F. R. (2009).Virtual microscopy in pathology education. Human Pathology 40(8), 1112-1121. Gagné, R. M. (1977). Task analysis. En L. J. Briggs (Ed.), Instructional Design: Principles and Application (pp. 123-150). Englewoods Cliffs, NJ: Educational Technology Publications.

García, R., Pérez,F. \& Talaya, I. (2008). Preferencias respecto a métodos instruccionales de los estudiantes universitarios de nuevo acceso y su relación con estilos de aprendizaje y estrategias motivacionales. Revista Electrónica de Investigación Psicoeducativa, 6(3), 547-570. Recuperado de: www.investigacionpsicopedagogica. org/ revista/new/ContadorArticulo.php?230

Hallet, D., Nunes, T. \& Bryant, P. (2010). Individual differences in conceptual and procedural knowledge when learning fractions. Postdoctoral research, University of Oxford. Journal of Educational Psychology, 102(2), 395-406. doi: 10.1037/a0017486. 
Hardy, M. W. (2008). A comparison of simulations and traditional laboratory exercises for student learning in secondary electronics instruction. (Disertación doctoral, Nova Southeastern University, United States, Florida). Recuperado de ProQuest Dissertations \& Theses: Nova Southeastern University Database. http://ezproxylocal. library.nova.edu/docview/304830531?accountid $=6579$

Lewis, D. (2008). The Acquisition of procedural skills: An analysis of the workedexample effect using animated demonstrations. (Disertación doctoral, Universidad de South Florida. http://ezproxylocal.library.nova.edu/docview/304461205?account id $=6579$

Liu, M. \& Reed, W. (1994). The Relationship between the learning strategies and learning styles in a hypermedia environment. Computers in Human Behavior, 10(4), 419-434.

Lo, J . \& Chan, C. (2008). Relationships between user cognitive styles and browsing behaviors of an online learning Web site. International Conference on Cyberworlds .pp 51-57. Recuperado de: http://www.computer.org/portal/web/csdl/doi/10.1109/ CW.2008.10 [2010, 10 de septiembre].

Mampadi, F., Chen, S., Ghinea, G. \& Chen, M. (2011). Design of adaptive hypermedia learning systems: A cognitive style approach. Computers \& Education 56 (2011), 10031011. Mayer, R. (2010) Applying the science of learning to medical education. Medical Education, 44, 543-549. Recuperado de: http://onlinelibrary.wiley.com/doi/10.1111/ j.1365-2923.2010.03624.x/pdf. [2010, 10 de septiembre].

Merrill, M. D. (2007). First principles of instruction: A synthesis. Trends and issues in instructional design and technology, (2nd ed.). Upper Saddle River, NJ : Prentice- Hall.

Patel, A. D., Gallagher, A. G., Nicholson, W. J . \& Cates, C. U. (2006). Learning curves and reliability measures for virtual reality simulation in the performance assessment of carotid angiography. Journal of the American College of Cardiology. 47(9), 1796-1802.

Pratt, J. A. (2000). Instruction in microbursts: The study of minimalist principles applied to online help. (Disertación doctoral. Universidad de Utah State). http:// ezproxylocal. library.nova.edu/docview $/ 304633287$ ?acco untid $=6579$

Reese, D. \& Tabachnick, B. (2010).The moment of learning: Quantitative analysis of exemplar gameplay supports CyGaMEs approach to embedded assessment. En The 2010 Annual Conference of the Society for Research on Educational Effectiveness. Washington, D. C. Recuperado de: http://www.sree.org/conferences/2010/program/ abstracts/191.pdf [2010, 15 de noviembre].

Simonson, M., Smaldino, S., Albright, M. \& Zvacek, S. (2006). Teaching and learning at a distance. Foundations of distance education (3a. ed.). Upper Saddle River, NJ : Prentice Hall. 


\section{Revista de investigaciones UNAD Volumen 10. Número 1. Junio 2011}

Slabicki, A. (2007). Using simulators to guide practice and reinforce online learning. (Dissertation doctoral, Universidad de Nova Southeastern, 2007). Recuperado de Dissertations \& Theses @ Nova Southeastern University Database. http:// ezproxylocal. library.nova.edu/docview /304719326/?accountid $=6579$

Weinstein, R. (2005). Innovations in medical imaging and virtual microscopy. Human Pathology, (36), 317-319.

White, G. \& Sivitanides, M. (2002). A theory of the relationships between cognitive requirements of computer programming languages and programmers' cognitive characteristics. Journal of Information Systems Education, 13(1) 59-66. 